Original Research Paper

\title{
Determination of Dynamic Viscosity of Viscous Oils using Mach Zehnder Digital Holographic Method
}

\author{
Rittirong Nuansri, Pichet Limsuwan and Prathan Buranasiri \\ Department of Physics, Faculty of Science, \\ King Mongkut's Institute of Technology Ladkrabang, Bangkok 10520, Thailand
}

\author{
Article history \\ Received: 04-06-2018 \\ Revised: $10-07-2018$ \\ Accepted: 21-07-2018 \\ Corresponding Author: \\ Rittirong Nuansri \\ Department of Physics, Faculty \\ of Science, King Mongkut's \\ Institute of Technology \\ Ladkrabang, Bangkok 10520, \\ Thailand \\ Email:kmitl.apl@gmail.com
}

\begin{abstract}
The digital in-line holography based on Mach Zehnder interferometer (MZDIH) was setup for the determination of dynamic viscosity of the viscous oils. The spherical irons were used as the falling objects in the viscous oils. The hologram images of the iron falling along its entire path were recorded as a video file on the CMOS camera. The recorded hologram images were reconstructed on the holoviewer program via MATLAB. Then, the diameter and velocity of the iron were obtained. Finally, the dynamic viscosity of viscous oils were determined and compared with standard values. From the experimental results, it can be concluded that the MZDIH method is the simple and precise tool for determining the dynamic viscosity of fluids.
\end{abstract}

Keywords: Holography, Dynamic Viscosity, Stokes Equation

\section{Introduction}

Since the holographic principle was explained by Dennis Gabor (Gabor, 1948), this technique has become an important optical method for research and industrial application. Producing a hologram requires two identical beams from a coherent light source, such as a laser. The first wave without distortion or non-diffracted light, is called the reference beam. The second beam, which passes through the object and the wavefronts of the light diffract caused by the object, is called the object beam. The hologram patterns are recorded of both phases and amplitudes on a photographic film. In Digital Holography (DH), since the conventional hologram consumes a lot of holographic recording films, the holographic recording media has been replaced by a Charged-Couple Device Detector (CCD) (Schnars and Jüptner, 1994). Generally, DH method is categorized into two kinds, on-axis (in-line) and off-axis configurations. The characteristics of the digital in-line geometry have the simplest setup. Here, the reference beam, object beam and the recording sensor are placed in the same axis. In the other configuration, the object and reference waves are at an angle with each other in order to avoid the noise incoming from the overlapping of both wavefronts (Micó et al., 2010). Currently, the DH method has found application in various fields because this technique can be determined both phases and amplitudes accurately and clearly in three dimensions (3D). For example, the plankton and other marine organisms as well as bacteria and fruit flies have been explored using the DH method (Sun et al., 2007; Xu et al., 2001). The density fluctuations of a standing wave in a thermoacoustic oscillation (Penelet et al., 2015) and the phase compensation of spherical beam (SánchezOrtiga et al., 2011) have been analyzed by the hologram patterns as well. The fingerprint patterns of human can also be observed and separated by investigating their profile intensity fringes (Plaipichit and Buranasiri, 2014). A number of previous researches have used the $\mathrm{DH}$ method for measuring the velocity of particles flowing through liquids in microchannel (Khatmullina and Isachenko, 2016), observing the flow and mechanics of fluid (Desse et al., 2012). These groups have also characterized the fluid's properties and adjusted the size and shape of objects traveling in the liquids (Murata and Yasuda, 2001; Darakis et al., 2009).

In this research, the kinematic viscosities of the four different lubricant oils used are 27.849, 52.050, 57.181 and 70.428 centi-Stokes (Measured at $40^{\circ} \mathrm{C}$ followed by ASTM D445). The lubricants used were supplied by the Goods and Exhibit Analysis Office, Excise Department of Thailand. The fluids used in this study are important lubricants typically used on engine parts in vehicles as well as in industrial machinery. Choosing a suitable oil allows machinery users to protect moving engine parts in the normal operation. However, the user has to know the oil's parameters before use, particularly, the density and viscosity. The viscosity value is the most important characteristic governing the behavior and grade 
classification of oils. There are various ways to measure the viscosity, such as a cold cranking simulator (CCS), which is used to measure the dynamic viscosity $(\eta$, in the unit of centipoise) and the kinematic viscosity ( $v$, in the unit of centistoke, cSt) following the ASTM D445 standard. These conventional methods are still accurate and precise; however, they have complicated operating procedures and use expensive equipment. Therefore, in our research, we propose an alternative way, Mach Zehnder digital in-line holography (MZDIH), to find out the dynamic viscosity ( $\eta$, in the unit of $\mathrm{mPa} . \mathrm{s})$ and kinematic viscosity ( $v$, in the unit of cSt.). This method is simpler, compact and less expensive than the conventional testing methods as mentioned above. In the experiment, the terminal velocity of a particle $\left(v_{t}\right)$ moving in different viscous lubricant oils contained in a liquid chamber was measured. Furthermore, the experimental values can then be used to determine the properties of the lubricant oils and also classify the commercial grade of the oils for their use in lubricating machine parts.

\section{Theoretical Backgrounds}

\section{Dynamic of a Particle in Fluids}

In non-compressible liquids, since the layers of the internal textures of the liquids have the relative activity to each other, the liquids have an internal resistant force. That force is called the viscous force or drag force, with direction opposite to the direction of particle motion. The drag force was determined by Sir George Stoke in 1845 (Collinson and Roper, 1995) and given by equation:

$$
F_{d}=6 \pi \eta r v_{t}
$$

where $\eta, r, v_{t}$ are dynamic viscosity, radius of the sphere and constant velocity, respectively. This law says that the force acting on a small spherical object, as it falls down through a viscous medium. Under the influence of the gravitational force:

$$
F_{g}=\rho_{0} V_{0} g
$$

where $\rho_{o}, V_{o}$ and $g$ are density of the object, volume of the spherical object and the gravitational constant, respectively. Due to the conditions of the object specified above, the Reynolds number in the system is small, therefore the flow of liquid remains laminar (Mott, 1994). The bouyant force as described by the principle of Archimedes (Serway and Jewett, 2014), is exerted on the object in the upward direction and given by:

$$
F_{B}=\rho_{f} V_{0} g
$$

When a small stationary spherical particle is released into a viscous liquid, the particle at a constant velocity $\left(v_{t}\right)$ experiences forces both upward and downward that must be in balance by Newton's 3rd law of motion. The dynamic viscosity can be expressed by equation:

$$
\eta=\left(\rho_{0}-\rho_{f}\right)-\frac{2 r^{2} g}{9 v_{t}}
$$

where $\rho_{0}, \rho_{f}, r$ and $\eta$ are object density, fluid density, radius of the spherical object and dynamic viscosity of the fluid, respectively.

Another force acting to a particle falling through the liquid in a small channel is the surface tension of the liquid in the channel. This force was recognized from the apparent stress in the surface layer of a liquid during its flow in a liquid chamber or between parallel plates.

Normally, the surface tension is an energy associated with an interface of liquid-liquid or liquid-air. Because the surface of liquids behaves like a membrane, the liquid surface may form a curvature between small channels, where the liquid drop can look more or less rounded (Hughes and Brighton, 1991). Therefore, the speed of the liquids at the center is maximum and the speed of liquid gradually decreases until it reaches a minimum at the liquid chamber surface. For this reason, the speed of a particle falling through a liquid contained in the liquid chamber is slower than moving through a liquid contained in a bigger one, because of the surface tension between the liquid and the container.

\section{Reconstruction Method of Digital Holography}

In this work, the principle of Fresnel diffraction and convolution theory of hologram reconstruction was described (Kim, 2011; Schnars and Jueptner, 2005). By assuming a parameter that propagates along the $\mathrm{z}$-axis to the sensor plane $(U(x, y))$ represents the two dimensional (2-D) diffraction of optical fields based on the HuygensFresnel principle. The diffraction of light through the optical filter in the rectangular coordinates can be shown as the optical diffraction equation:

$U(x, y)=\frac{z}{j \lambda} \iint O(\xi, \eta) \frac{\exp (j k r)}{r^{2}} d \xi d \eta$

where $O(\xi, \eta)$ represents an optical field in two dimensions at the object plane, $r$ equals to $\left[z^{2}+(x-\xi)^{2}+\right.$ $\left.(y-\eta)^{2}\right]^{1 / 2}$, which is the displacement from any point on the object plane $(O(\xi, \eta))$ to the recording or sensor plane $(x, y)$ and $z$ is the propagation distance of the optical field as shown in Fig. 1. The hologram function can be expressed using the convolution technique as illustrated in Equation 6: 


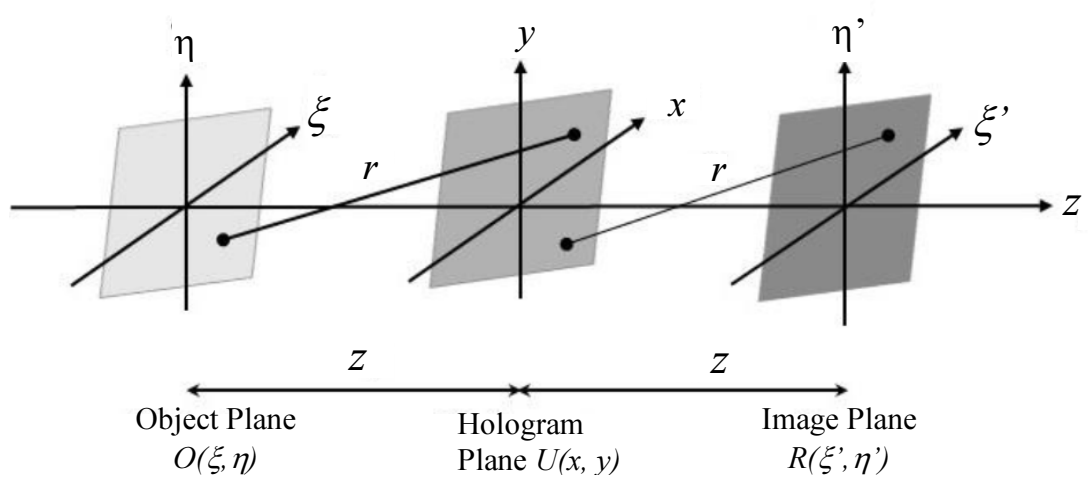

Fig. 1: A coordinate system for the numerical reconstruction of recorded images by digital holographic technique

$U(x, y)=F^{-1}\{F[O(\xi, \eta)] . F[\zeta, \eta, x, y]\}$

where the Kernel's equation is given by $\mathrm{g}(\zeta, \eta, \mathrm{x}, \mathrm{y})=\frac{z}{j \lambda} \frac{\exp (j k r)}{r^{2}}$ and $k \quad$ represents the wavenumber of light, which equals $2 \pi / \lambda$. Because the size of the recording sensor is very small compared to the distance of light propagation, $r^{2}$ is reduced to $z^{2}$. Hence, this equation can be rewritten as $\mathrm{g}(\zeta, \eta, \mathrm{x}, \mathrm{y})=\frac{\exp (j k r)}{j k z}$. By utilizing the inverse Fourier transform of the filtered Huygens-Fresnel principle, the optical field at the image plane $\left(R\left(\xi^{\prime}, \eta^{\prime}\right)\right)$ can be reconstructed as:

$$
R\left(\xi^{\prime}, \eta^{\prime}\right)=F^{-1}\{F[O(x, y)] . F[g(\zeta, \eta, x, y)]\}
$$

The holograms recorded by the sensor are transferred to a computer and reconstructed based on the HuygensFresnel principle as shown in the Equation 7.

\section{Experimental Method}

\section{Experimental Setup}

Fig. 2(a) shows the Mach Zehnder Digital Holographic (MZDH) system used for measuring the dynamic viscosity of a spherical iron object travelling in the viscous oil.

In the MZDH system, the light source was a laser diode with $635 \mathrm{~nm}$ wavelength at a power of $1 \mathrm{~mW}$ (LDM115G/633/1). A convex lens $\mathrm{L}_{1}, f_{1}=200 \mathrm{~mm}$, was used to collimate the laser beam that propagates in the z-axis from the light source. Then, the laser beam of about $20 \mathrm{~mm}$ in diameter was separated into two identical beams, the reference beam and the object beam, by a beam splitter $\mathrm{BS}_{1}$. The reference beam, without any distortion, is reflected at mirror $\mathrm{M}_{1}$, while the object beam is reflected at mirror $\mathrm{M}_{2}$.

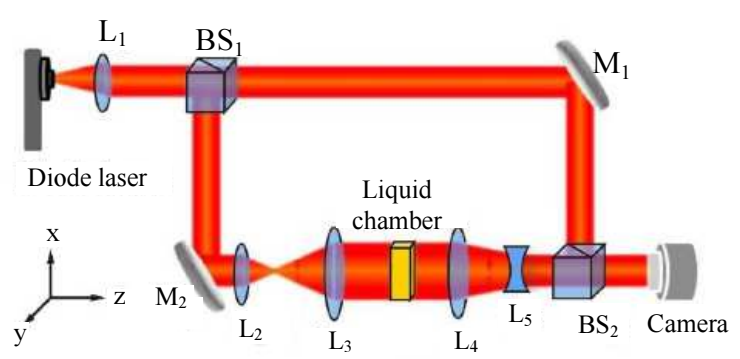

(a)

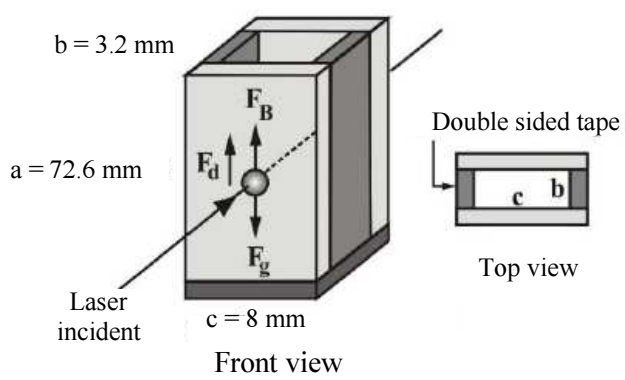

(b)

Fig. 2: (a) Schematic diagram of digital in-line holographic setup (DIH) based on Mach Zehnder interferometer and (b) the forces acting on the object moving in the viscous liquid contained in the liquid chamber

The object beam was a short focal length convex lens $\mathrm{L}_{2}, f_{2}=10 \mathrm{~mm}$ and a long focal length expanded convex lens $\mathrm{L}_{3}$ and $\mathrm{L}_{4}, f_{3,4}=200 \mathrm{~mm}$ to cover the whole volume of liquid chamber. Then, the transmitted beam passed through the object was collimated again by concave lens $\mathrm{L}_{5}, f_{5}=-200 \mathrm{~mm}$ to generate the parallel beam. Finally, the two beams were recombined at the beam splitter $\mathrm{BS}_{2}$ and the hologram patterns were recorded on the CMOS camera (Canon EOS $700 \mathrm{D}$, Japan, $5184 \times 3456$ pixels, $4.29 \mu \mathrm{m}$. pixel pitch). 
The numerically reconstructed distance $(\mathrm{R})$ based on the Huygens-Fresnel principle as mentioned in section 2.2 was found to be $400 \mathrm{~mm}$.

\section{Materials and Sample Preparation}

Three spherical irons, each one with a diameter of about $1.50 \mathrm{~mm}$ and a density of $7.00 \mathrm{~g} / \mathrm{cm}^{3}$ were used as the object. The object size was measured by a digital Vernier caliper with $0.01 \mathrm{~mm}$ resolution. The average diameter of the object was found to be $1.50 \pm 0.02 \mathrm{~mm}$.

The liquid chamber was prepared by using two glass slides, each one having a dimension (length $\mathrm{x}$ width $\mathrm{x}$ thickness) of $76.2 \times 25.4 \times 1.5 \mathrm{~mm}^{3}$ (SAIL, No. 7101). They were attached with two layers of double sided tape foam with $3.2 \mathrm{~mm}$ thick (3M, 4026 Natural PUR Foam) between the slides and sealed at the bottom by hot melted adhesion glue. Figure $2 b$ shows the diagram of liquid chamber with a volume $(\mathrm{a} \times \mathrm{b} \times \mathrm{c})$ of $72.6 \times 3.2 \times 8 \mathrm{~mm}^{3}$.

The viscous oils used in this study were supplied by the Goods and Exhibit Analysis Office, Excise Department of Thailand. The properties of the oils are given in Table 1 . The values of density $\left(\rho_{f}\right)$ at $15^{\circ} \mathrm{C}$ and the kinematic viscosity $(v)$ at $40^{\circ} \mathrm{C}$ were obtained from ASTM D1298 and ASTM D445, respectively.

Table 1: Properties of the viscous oils used in the experiment

\begin{tabular}{lll}
\hline Oils & $\begin{array}{l}\text { Density at } \\
15^{\circ} \mathrm{C}\left(\mathrm{g} / \mathrm{cm}^{3}\right)\end{array}$ & $\begin{array}{l}\text { Kinematic viscosity } \\
\text { at } 40^{\circ} \mathrm{C}(\mathrm{cSt} .)\end{array}$ \\
\hline Lubricant oil (ZE-PRO32) & 0.87154 & 27.849 \\
Transformer oil (FR-3) & 0.92478 & 52.050 \\
Gasoline engine oil & 0.87398 & 57.181 \\
Cold lubricant oil & 0.95870 & 70.428 \\
\hline
\end{tabular}

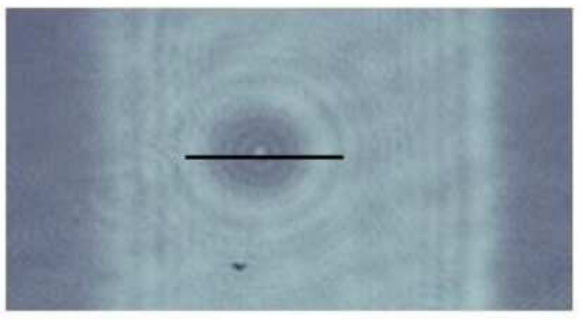

(a)

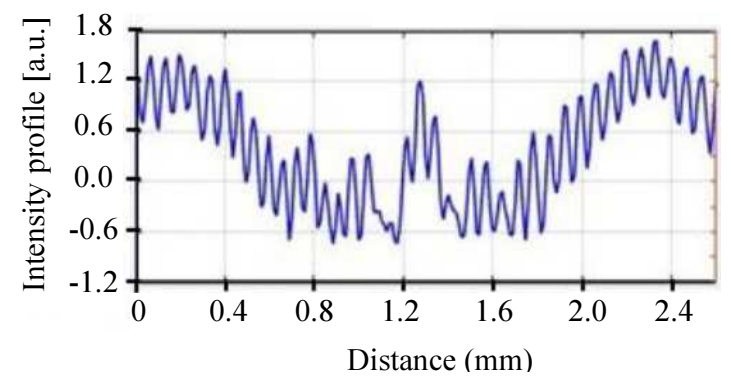

(c)

\section{Results and Discussion}

\section{Diameter Measurement of Spherical Iron}

Among four oils used in this work, the lubricant oil is transparent. Therefore, it was used for the diameter measurement of spherical iron. It was filled in the liquid chamber which placed in the object arm of the interferometer as shown in Fig. 2(a). The first spherical iron object was dropped into the liquid chamber. The hologram images of the object falling along its entire path were recorded as a video file on the CMOS camera (Canon EOS 700D, Japan, 5184×3456 pixels, $4.29 \mu \mathrm{m}$. pixel pitch). Then, the recorded hologram images were reconstructed on the holoviewer program via MATLAB by using the reconstructing distance of $400 \mathrm{~mm}$. Figs. 3(a) and 3(b) show the typical recorded hologram images and the reconstructed images of a falling spherical iron at any position in the lubricant oil. However, it is noted that all the experiments in this work were performed at the room temperature $\left(27^{\circ} \mathrm{C}\right)$.

The holoviewer program via MATLAB was used to analyze the image as shown in Figure 3(d) and the diameter was determined. The experiments were carried for three times and the results are given in Table 2 . The average diameter of the first spherical iron was found to be $1.51251 \mathrm{~mm}$. The similar experiments were performed for the second and third spherical irons and the results are also given in Table 2. It is seen that the diameter of spherical iron obtained by DIH method agree with that measured by the Vernier caliper. In addition, it should be pointed out that DIH method can be used for diameter measurement with very high accuracy ( 5 decimal points).

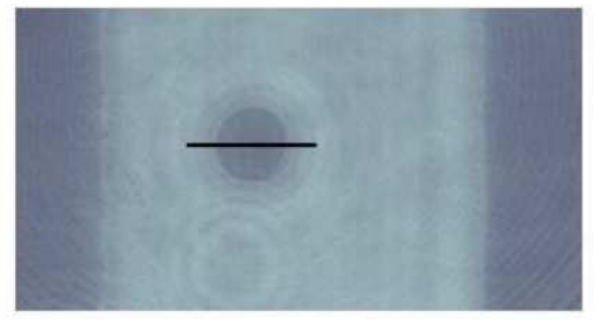

(b)

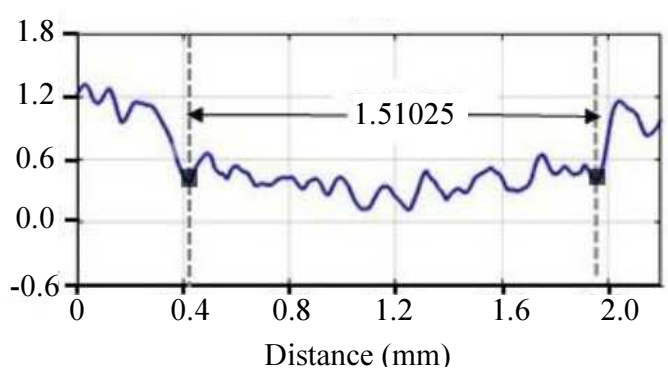

(d)

Fig. 3: (a) Recorded hologram images, (b) reconstructed images, (c) intensity profile of recorded hologram images and (d) intensity profile of reconstructed images of a falling spherical iron in the lubricant oil 


\section{Determination of Spherical Iron Velocity}

To determine the velocity of spherical irons, each one was dropped into the liquid chamber and the hologram images were recorded as a video file on the CMOS camera (Canon, EOS 700D, Japan, $5184 \times 3456$ pixels, $4.29 \mu \mathrm{m}$. pixel pitch). The video files of a spherical iron falling in the liquid chamber were captured as an image at different positions $(\mathrm{P})$ along the liquid chamber of 10 , $20,30,40,50$ and $60 \mathrm{~mm}$ as denoted by $\mathrm{P}_{10}, \mathrm{P}_{20}, \ldots, \mathrm{P}_{60}$, respectively. Then, the falling times $(\mathrm{t})$ at any measured position were obtained and the velocity of spherical iron was determined. Figs. 4(a) and 4(b) show the recorded and reconstructed hologram images of a spherical iron falling in liquid chamber filled with the lubricant oil. The typical values of measured velocity and time at different positions of three spherical irons falling in the lubricant oil are given in Table 3.

Similar experiments were performed with transformer oil, gasoline engine oil and cold lubricant oil but the results are not given here. However, the relationship between velocity and distance for all spherical irons in lubricant oil, transformer oil, gasoline engine oil and cold lubricant oil are illustrated in Fig. 5. It is clearly observed that the velocity is constant in the falling path for all spherical irons in all viscous oils. Hence, the average velocity values are assumed to be terminal velocity for spherical irons which will be used for the determination of dynamic viscosity as shown in Table 4.

\section{Determination of Dynamic Viscosity of the Oils}

The dynamic viscosity of the oils was determined from Equation (4):

$$
\eta=\left(\rho_{0}-\rho_{f}\right) \frac{2}{9} \frac{r^{2} g}{v_{t}}
$$

where:

$\eta=$ Dynamic viscosity of the oil $(\mathrm{kg} / \mathrm{m} . \mathrm{s})$

$\rho_{o}=$ Density of the iron $=7000 \mathrm{~kg} / \mathrm{m}^{3}$

$\rho_{f}=$ Density of the oil (obtained from Table 1)

$r=$ Radius of the iron (obtained from Table 2)

$g=$ Gravitational constant $=9.80665 \mathrm{~m} / \mathrm{s}^{2}$

$v_{\mathrm{t}}=$ Terminal velocity of an iron falling in the oil (obtained from Table 3) (a)
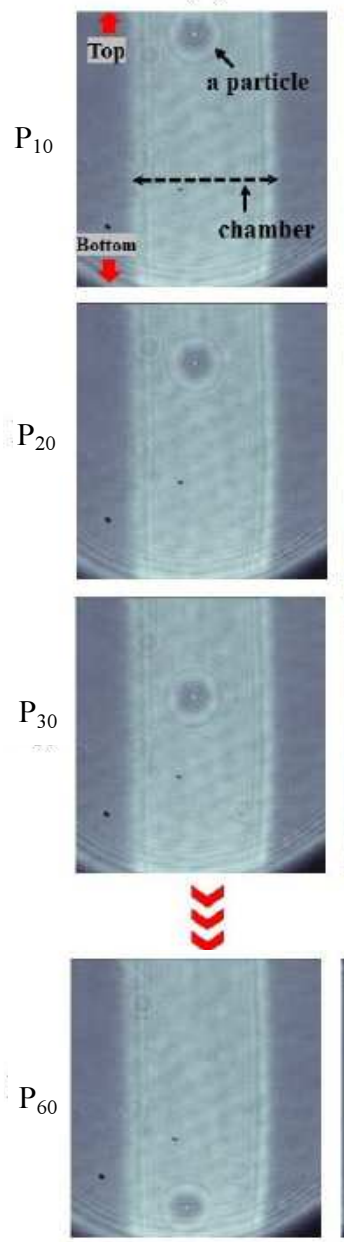

(b)
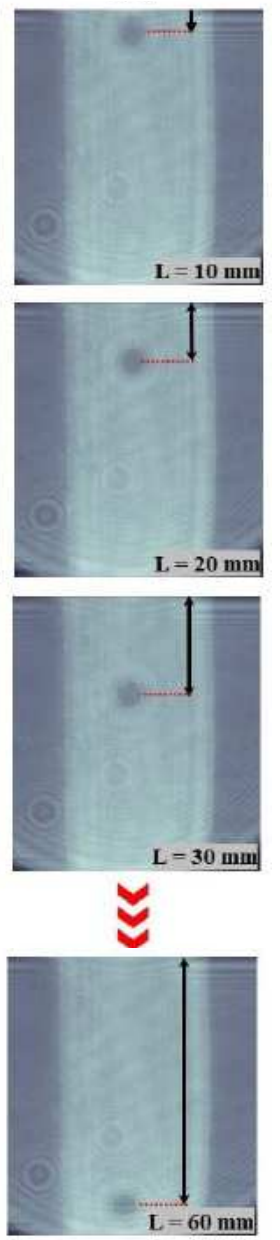

Fig. 4: (a) Recorded hologram images and (b) reconstructed images showing the position of a spherical iron falling in the lubricant oil

Table 2: Measured diameter of spherical irons

\begin{tabular}{|c|c|c|c|}
\hline \multirow[b]{2}{*}{ No. } & \multicolumn{3}{|c|}{ Diameter (mm) } \\
\hline & $1^{\text {st }}$ iron & $2^{\text {nd }}$ iron & $3^{\text {rd }}$ iron \\
\hline 1 & 1.51025 & 1.49987 & 1.52108 \\
\hline 2 & 1.51509 & 1.49891 & 1.52315 \\
\hline 3 & 1.51218 & 1.50006 & 1.51057 \\
\hline Average & 1.51251 & 1.49961 & 1.51827 \\
\hline
\end{tabular}

Table 3: Measured velocity and time at different positions of spherical irons falling in the lubricant oil

\begin{tabular}{|c|c|c|c|c|c|c|}
\hline \multirow{2}{*}{$\begin{array}{l}\text { Position } \\
(\mathrm{mm})\end{array}$} & \multicolumn{2}{|c|}{$1^{\text {st }}$ iron } & \multicolumn{2}{|c|}{$2^{\text {nd }}$ iron } & \multicolumn{2}{|c|}{$3^{\text {rd }}$ iron } \\
\hline & $t(\mathrm{~s})$ & $v(\mathrm{~mm} / \mathrm{s})$ & $t(\mathrm{~s})$ & $v(\mathrm{~mm} / \mathrm{s})$ & $t(\mathrm{~s})$ & $v(\mathrm{~mm} / \mathrm{s})$ \\
\hline 10 & 0.042 & 237.520 & 0.042 & 236.952 & 0.042 & 237.025 \\
\hline 20 & 0.084 & 238.875 & 0.085 & 235.395 & 0.085 & 236.520 \\
\hline 30 & 0.125 & 240.168 & 0.128 & 235.051 & 0.125 & 239.541 \\
\hline 40 & 0.167 & 240.010 & 0.172 & 232.982 & 0.169 & 236.851 \\
\hline 50 & 0.209 & 238.996 & 0.214 & 234.085 & 0.209 & 239.021 \\
\hline 60 & 0.250 & 239.982 & 0.254 & 236.541 & 0.252 & 237.658 \\
\hline Average & & 239.259 & & 235.168 & & 237.769 \\
\hline
\end{tabular}


Table 4: Dynamic viscosity $(\eta)$ and kinematic viscosity $(v)$ of four viscous oils.

\begin{tabular}{|c|c|c|c|c|}
\hline \multirow[b]{2}{*}{ Parameters } & \multicolumn{4}{|l|}{ Oils } \\
\hline & Lubricant oil & Transformer oil & Gasoline engine oil & Cold lubricant oil \\
\hline $1^{\text {st }}$ iron & Average radius $=0.75626 \mathrm{~mm}$ & & & \\
\hline$v_{t}(\mathrm{~mm} / \mathrm{s})$ & 239.259 & 149.721 & 129.911 & 99.064 \\
\hline$\eta(\mathrm{mPa} . \mathrm{s})$ & 31.925 & 50.574 & 58.773 & 76.008 \\
\hline$v(\mathrm{cSt})$ & 36.630 & 54.687 & 67.247 & 79.282 \\
\hline $2^{\text {nd }}$ iron & Average radius $=0.74981 \mathrm{~mm}$ & & & \\
\hline$v_{t}(\mathrm{~mm} / \mathrm{s})$ & 235.168 & 146.641 & 124.933 & 95.008 \\
\hline$\eta(\mathrm{mPa} . \mathrm{s})$ & 31.928 & 50.759 & 60.077 & 77.907 \\
\hline$v(\mathrm{cSt})$ & 36.635 & 54.887 & 68.739 & 81.263 \\
\hline $3^{\text {rd }}$ iron & Average radius $=0.75914 \mathrm{~mm}$ & & & \\
\hline$v_{t}(\mathrm{~mm} / \mathrm{s})$ & 237.769 & 152.961 & 130.904 & 100.438 \\
\hline$\eta(\mathrm{mPa} . \mathrm{s})$ & 32.370 & 49.880 & 58.772 & 75.540 \\
\hline$v(\mathrm{cSt})$ & 37.141 & 53.937 & 67.247 & 78.794 \\
\hline
\end{tabular}

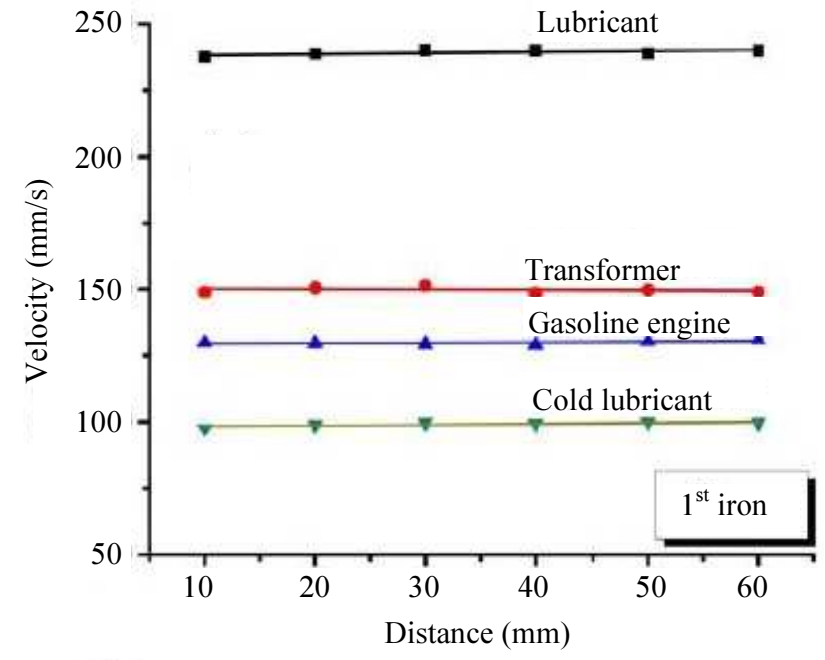

(a)

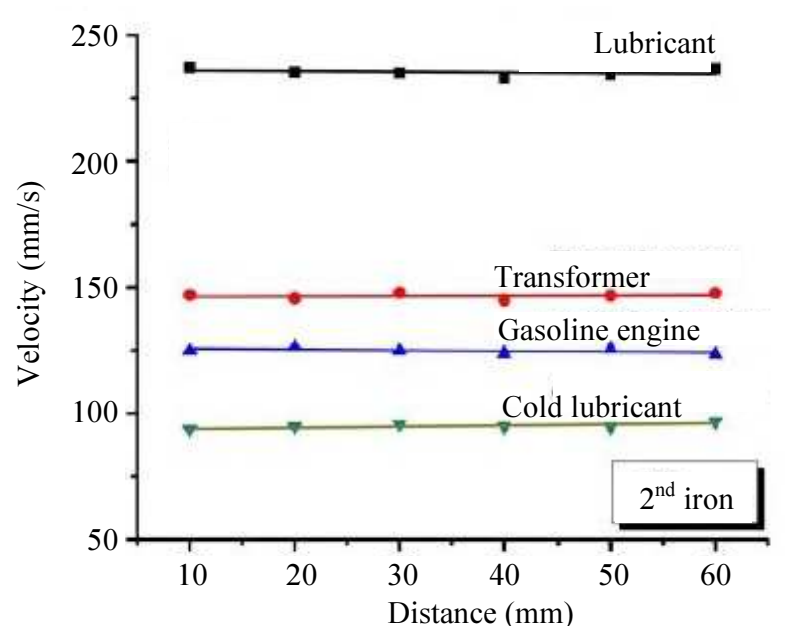

(b)

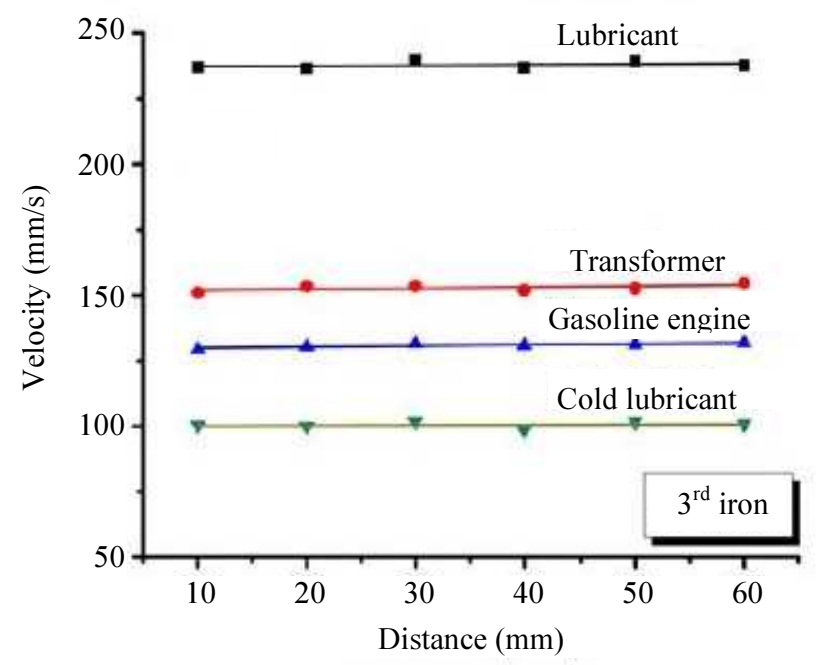

(c)

Fig. 5: Variation of spherical iron velocity with falling distance of: (a) first irons, (b) second iron and (c) third iron in four different viscous oils 
Then, the dynamic viscosity of all four viscous oils using three spherical irons were determined and the results are given Table 4 .

The relationship between dynamic viscosity $(\eta$, in mPa.s unit) and kinematic viscosity ( $v$, in cSt unit) is given by:

$$
\eta=\rho_{f} v \times 10^{-3}
$$

Therefore, the kinematic viscosity of all four viscous oils were calculated and the results are given Table 4.

From the dynamic viscosity $(\eta)$ given in Table 1 , for example the lubricant oil, the $\eta$ as obtained from three spherical irons are $31.925,31.928$ and $32.370 \mathrm{mPa} . \mathrm{s}$, respectively, which are in good agreement. The dynamic viscosity for lubricant oil, transformer oil, gasoline engine oil and cold lubricant oil are approximately 31.925, $50.574,58.773$ and $76.008 \mathrm{mPa} . \mathrm{s}$, respectively. Therefore, the cold lubricant oil gives the highest dynamic viscosity.

To compare the results obtained in this work with those of standard values of ASTMD445 as given Table 1, it is necessary to convert the dynamic viscosity $(\eta)$ into the kinematic viscosity $(v)$ by Equation (7) as mentioned above. The kinematic viscosity $(v)$ of all four viscous oils obtained from three spherical irons are displayed in Fig. 6.

From Fig. 6, it is clearly observed that the kinematic viscosity of all four viscous oils obtained from the first, second and third spherical irons are approximately equal.

Furthermore, it is seen that the kinematic viscosity values obtained in this work are higher than those of standard values as given in Table 1. The difference in kinematic viscosity values are due to the temperature difference of the viscous oils. Since all the experiments in this work were performed at $27^{\circ} \mathrm{C}$ while the standard measurements were carried out at $40^{\circ} \mathrm{C}$. However, it is observed that the higher viscous oil temperature results in the lower kinematic viscosity.

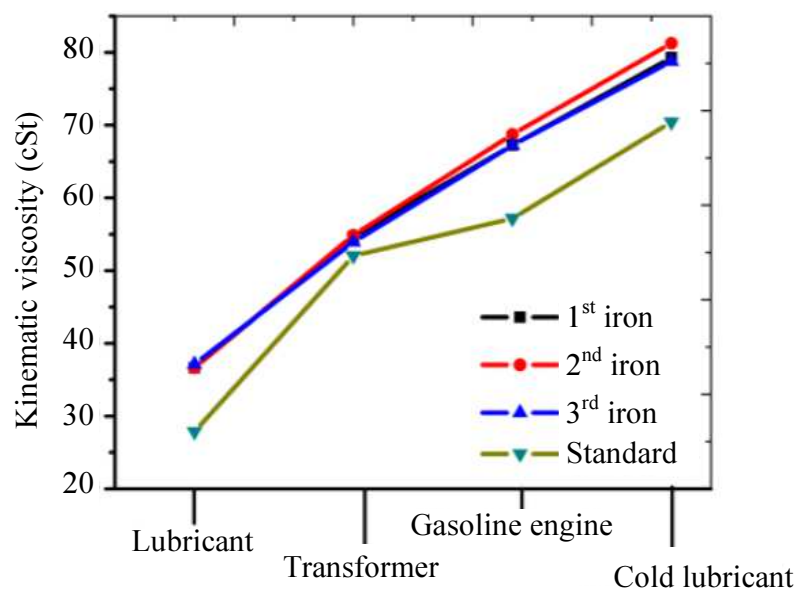

Fig. 6: Comparing the kinematic viscosity between the experimental results and standard values for four viscous oils

\section{Conclusion}

The digital in-line holographic based on Mach Zehnder interferometer was setup for the determination of dynamic viscosity of four viscous oils. In the system, the liquid chamber filled with the viscous oil to be studied was developed. Three spherical irons of about the same diameter were used as the falling objects in the liquid chamber. The hologram images of the spherical iron falling along its entire path were recorded as a video file on the CMOS camera. The recorded hologram images were reconstructed on the holoviewer program via MATLAB. Then, the diameter and velocity of the spherical iron were obtained. Finally, the dynamic viscosity $(\eta)$ of all four viscous oils were determined. It can be concluded that this method is the simple and precise tool for the determination of dynamic viscosity of fluids.

\section{Acknowledgement}

Rittirong Nuansri would like to thank Dr. Witoon Yindeesuk, Head of Physics Department for providing all facilities to make this work complete. This article has been supported the reconstructed tool from Department of Physics, Michigan Technological University, U.S.A.

\section{Funding Information}

This work was financially supported by the Department of Physics, King Mongkut's Institute of Technology Ladkrabang.

\section{Author's Contributions}

Rittirong Nuansri: Carried out the experimental setup, collected all the measurement data and prepared the original manuscript.

Pichet Limsuwan: Helped in writing the manuscript.

Prathan Buranasiri: Read and approved the manuscript.

\section{Ethics}

This article is original and contains unpublished material. It is confirmed that all authors have read and approved the manuscript and there are no ethical issues involved.

\section{References}

Collinson, C. and T. Roper, 1995. Particle Mechanics. Elsevier Ltd., Oxford, pp: 30. ISBN: 0-340-61046-8.

Darakis, E., T. Khanam, A. Rajendran, V. Kariwala and T.J. Naughton et al., 2009. Microparticle characterization using digital holography. Chemical Eng. Sci., 65: 1037-1044.

DOI: $10.1016 /$ j.ces.2009.09.057 
Desse, J.M., P. Picart and P. Tankam, 2011. Digital color holography applied to fluid and structural mechanics. Optics Lasers Engineering, 50: 18-28. DOI: $10.1016 /$ j.optlaseng.2011.06.018

Gabor, D., 1948. A new microscopic principle. Nature, 161: 777-778. DOI: 10.1038/161777a0

Hughes, W.F. and J.A. Brighton, 1991. Theory and Problems of Fluid Dynamics. McGraw-Hill, Inc., Singapore, pp: 5. ISBN: 0-07-031117-x

Khatmullina, L. and I. Isachenko, 2016. Settling velocity of microparticles of regular shapes. Marine Pollution Bulletin, 114: 87-880.

DOI: 10.1016/j.marpolbul.2016.11.024

Kim, M.K., 2011. Digital Holographic Microscopy. Springer, New York, USA, ISBN-10: 978-1-4419-7792-2, pp: 47-50.

Micó, V., C. Ferreira, Z. Zalevsky and J. García, 2010. Basic principles and applications of digital holographic microscopy. Microscopy: Science, Technology, Applications and Education, pp: 1411-1418.

Mott, R.L., 1994. Applied Fluid Mechanics. 4th Edn., Macmillan Publishing Company, New York, ISBN-10: 0-02-384231-8, pp: 477.

Murata, S. and N. Yasuda, 2001. Potential of digital holography in particle measurement. Optics Laser Technology, 32: 567-574.

Penelet, G., M. Leclercq, T. Wassereau and P. Picart, 2015. Measurement of density fluctuations using digital holographic interferometry in a standing wave thermoacoustic oscillator. Experimental Thermal Fluid Science, 70: 176-184.

DOI: 10.1016/j.expthermflusci.2015.09.012
Plaipichit, S. and P. Buranasiri, 2014. Moisture effect of fingerprint using total internal reflection digital inline holography. Optical Engineering, 53: 112315. DOI: $10.1117 / 1 . O E .53 .11 .112315$

Sánchez-Ortiga, E., P. Ferraro, M. Martínez-Corral, G. Saavedra and A. Doblas, 2011. Digital holographic microscopy with pure-optical spherical phase compensation. Optical Society America, 28: 1410-1417. DOI: $10.1364 /$ JOSAA.28.001410

Schnars, U. and W. Jueptner, 2005. Digital Holography. Springer Berlin Heidelberg, New York, ISBN-10: 3-540-21934-x, pp: 44-54.

Schnars, U. and W. Jüptners, 1994. Direct recording of holograms by a CCD target and numerical reconstruction. Applied Optics, 33: 179-181.

Serway, R.A. and J.W. Jewett, 2014. Physics for Scientists and Engineers with Modern Physics. 9th Edn., CENGAGE LEARNING, USA, ISBN-13: 978-1-133-95399-9, pp: 423-425.

Sun, H., D.C. Hendry, M.A. Player and J. Watson, 2007. In situ underwater electronic holographic camera for studies of plankton. IEEE J. Oceanic Engineering, 32: 373-382. DOI: 10.1109/JOE.2007.891891

$\mathrm{Xu}$, W., M.H. Jericho, I.A. Meinertzhagen and H.J. Kreuzer, 2001. Digital in-line holography for biological applications. PNAS 98: 11301-11305. DOI: $10.1073 /$ pnas. 191361398 